\title{
Chaotic dynamics of a pulse modulated control system for a heating unit
}

\author{
Yuriy A. Gol'tsov ${ }^{1, *}$, Alexander S. Kizhuk ${ }^{1}$, and Vasiliy G. Rubanov ${ }^{1}$ \\ ${ }^{1}$ Department of Engineering Cybernetics, Belgorod State Technological University n.a. \\ V.G. Shukhov, Kostyukov Str., 46, 308012, Belgorod, Russia
}

\begin{abstract}
The dynamic modes and bifurcations in a pulse control system of a heating unit, the condition of which is described through differential equations with discontinuous right-hand sides, have been studied. It has been shown that the system under research can demonstrate a great variety of nonlinear phenomena and bifurcation transitions, such as quasiperiodicity, multistable behaviour, chaotization of oscillations through a classical period-doubling bifurcations cascade and bordercollision bifurcation.
\end{abstract}

\section{Introduction}

The technology of monocrystals growth is a controlled crystallization process, during which the quality of a growing crystal is determined by the accuracy of controlling phase transition conditions $[1,2]$. When growing a sapphire crystal it is necessary to provide the temperature change pattern in the crucible from $25{ }^{\circ} \mathrm{C}$ to $2050{ }^{\circ} \mathrm{C}$ with a certain degree of its increase and decrease, which implies the application of the automated control system with the function of the software setting of the temperature variation in the crucible with the required accuracy.

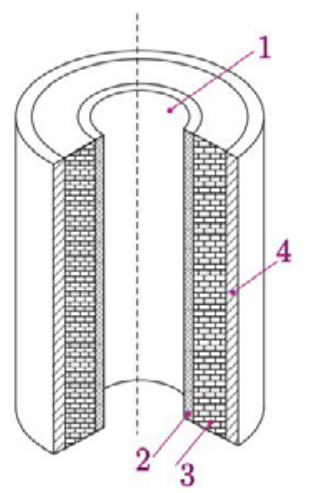

Fig. 1. Schematic diagram of the heating unit.

\footnotetext{
*Corresponding author: umin@mail.ru
} 
The thermal facility, i.e. the heating unit, consists of the following areas: internal furnace space 1 , filled with air or gas, nichrome electric heater 2 , uniformly located in the inner layer of the lining 3, made of magnesite bricks, and the outer layer of the lining 4, made of the mineral wool, in a cylindrical beaker made of galvanized steel (fig. 1). The geometric shape of the furnace is a bounded cylinder, with the lining on its top and bottom.

To solve the synthesis problem of the control law, using the classical approximation method and the shareware FOMCON library, based on experimental transient response curve of a heating object we have defined the following transfer function of the heating unit:

$$
W(s) \frac{K}{\left(T_{1} \cdot s+1\right)\left(T_{2} \cdot s+1\right)},
$$

where $K$ - transfer factor of an object, and $T_{1}, T_{2}$ - time constants of an object [3].

The currently used temperature regulators with thyristor converters distort considerably the input current curve shape, which results in the appearance of non-sinusoidal modes in the supply network.

In order to eliminate these drawbacks a high-power heating unit control system, based on high-frequency switching power electronics converter with pulse-width modulation, has been developed and implemented on the basis of the authors' patent [4]. The improvement of the energy performance together with the simplification of the production facility control is achieved by applying a field transistors converter as a key element with using fractional control laws of pulse-width modulation, which improves the system's quality [5].

But at the variations of the controlled objects' parameters in nonlinear pulse systems, as well as under the external disturbances, the complicated nonlinear phenomena can occur, including high-frequency oscillations, multiple of the modulation frequency, and quasiperiodic or chaotic modes [6-8].

The appearance of such oscillatory modes leads not only to the reduction of the control accuracy, but also to sudden breakdown of the technological equipment.

The purpose of this paper is the numerical study of bifurcation phenomena in the pulse modulated control system of a high-power heating unit.

The paper is organized as follows. In section 2 we describe a model of the heating unit with pulse-width modulated control. The behaviour of such a system may be represented by a two-dimensional piecewise-smooth set of nonautonomous differential equations. We reduce the investigation of this system to studying the dynamics of a two-dimensional piecewise-smooth map.

In section 3, we discuss the bifurcation phenomena occurring in the considered system. The obtained results are summarized in Conclusions.

\section{The task statement and analytical solution}

The motion equation of a heating unit control system, the continuous linear part of which is described by the transfer function (1), is as follows:

$$
T_{1} T_{2} \frac{d^{2} x}{d t^{2}}+\left(T_{1}+T_{2}\right) \frac{d x}{d t}+x=K U \varphi(\varepsilon)
$$

where $x$-is the temperature in the heating unit, $\varepsilon$ and $\varphi(\varepsilon)$ - are the input (error) and output signals of the pulse-width modulator, respectively; $K$ - transfer factor of the continuous linear part; $T_{1}, T_{2}$ - time constants.

Let us introduce the state variables 


$$
x=x_{1}, \frac{d x}{d t}=x_{2} .
$$

Then, the closed-loop system expressed by (2) can now be rewritten as a set of two coupled ordinary differential equations:

$$
\frac{d x_{1}}{d t}=x_{2}, \frac{d x_{2}}{d t}=-\frac{1}{T_{1} T_{2}} x_{1}-\frac{T_{1}+T_{2}}{T_{1} T_{2}} x_{2}+\frac{K U}{T_{1} T_{2}} \varphi(\varepsilon) .
$$

The switching function $\varphi(\varepsilon)$ (output signal of the modulator) is defined as:

$$
\begin{gathered}
\varphi(\varepsilon)= \begin{cases}1, & k \cdot T_{0}<t<k \cdot T_{0}+\tau_{k} ; \\
0, & k \cdot T_{0}+\tau_{k}<t<(k+1) \cdot T_{0} ;\end{cases} \\
k=0,1,2, \ldots
\end{gathered}
$$

Here $T_{0}$ is the period of the ramp signal and $\tau_{k}$ is the pulse duration within the time interval $\left.k \cdot T<t<(k+1) \cdot T_{0}\right)$.

In the present analysis we shall consider a system with the proportional feedback corrector and pulse-width modulation of the first type (see, for example, [6]).

In such a system, depending on the error signal $\varepsilon$ the value of $\tau_{k}$ is determined as:

$$
\tau_{k}=\left\{\begin{array}{cl}
0, & \varepsilon<0 \\
T_{0}, & \varepsilon>V_{s} ; \\
\frac{T_{0} \varepsilon}{V_{s}}, & 0 \leq \varepsilon \leq V_{s},
\end{array}\right.
$$

where

$$
\varepsilon=\alpha\left(V_{r e f}-\beta x_{1}\left(k T_{0}\right)\right)
$$

Here $\alpha$ and Vref are the amplification constant and reference signal. $V s$ represents the amplitude of the rump signal and $\beta$ is the sensitivity of the temperature sensor. The mathematical model (3) of such a system may be cast into the form:

$$
\frac{d X}{d t}=\left\{\begin{array}{ccc}
A X+B, & \text { if } & \xi(t, X)>0 \\
A X, & \text { if } & \xi(t, X)<0
\end{array}\right.
$$

with

$$
X=\left[\begin{array}{l}
x_{1} \\
x_{2}
\end{array}\right], A=\left[\begin{array}{cc}
0 & 1 \\
-\frac{1}{T_{1} T_{2}} & -\frac{T_{2}+T_{2}}{T_{1} T_{2}}
\end{array}\right], B=\left[\begin{array}{c}
0 \\
\frac{K U}{T_{1} T_{2}}
\end{array}\right]
$$

and

$$
\xi(t, X)=V_{r e f}-\beta \cdot x_{1}\left(k \cdot T_{0}\right)-\frac{V_{s}}{\alpha}\left(t / T_{0}-\left\lfloor t / T_{0}\right\rfloor\right)
$$


Here the number $\left\lfloor t / T_{0}\right\rfloor$ denotes the largest integer not greater than $t / T_{0}$ (i.e., the integer part, or floor, of $\left.t / T_{0}\right)$. The scalar function $\xi(t, X)$ determines the switching manifold

$$
\Sigma=\{(t, X): \xi(t, X)=0\}
$$

that separates the state space into two different regions

$$
\Sigma_{+}=\{(t, X): \xi(t, X)>0\}, \Sigma_{-}=\{(t, X): \xi(t, X)<0\}
$$

in which the dynamic behaviour of the system (5) is governed by different vector fields. It is easy to show that any solution of (5) will intersect the switching manifold (or discontinuity boundary) $\Sigma$ transversely (see [8]).

The investigation of the dynamical system (5) has been reduced to the study of the twodimensional stroboscopic map.

Within the domain

$$
k T_{0}<t<t_{k}, t_{k}=k T_{0}+\tau_{k},
$$

the system (5) has the form

$$
\frac{d X}{d t}=A X+B, \quad X\left(k \cdot T_{0}\right)=X_{k}
$$

with solution

$$
X(t)=\exp \left(A\left(t-k \cdot T_{0}\right)\left[X_{k}+D\right]-D .\right.
$$

At the switching time $t=t_{k}$ :

$$
\begin{gathered}
X\left(t_{k}\right)=\exp \left(A\left(t_{k}-k \cdot T_{0}\right)\left[X_{k}+D\right]-D,\right. \\
D=A^{-1} B .
\end{gathered}
$$

In the subsequent time interval

$$
t_{k}<t<(k+1) T_{0},
$$

system (5) takes the form

$$
\frac{d X}{d t}=A X
$$

with the solution

$$
X(t)=\exp \left(A\left(t-t_{k}\right)\right) X\left(t_{k}\right) .
$$

In this way, for $t=(k+1) \cdot T_{0}$

$$
\begin{gathered}
X_{k+1}=\exp \left(A\left((k+1) \cdot T_{0}-t_{k}\right)\right) X\left(t_{k}\right), \\
X_{k+1}=X\left((k+1) \cdot T_{0}\right)
\end{gathered}
$$


Substituting the expression for $X\left(t_{k}\right)$ into (6), we obtain the two-dimensional piecewise-smooth map [6]

$$
\begin{gathered}
X_{k+1}=\exp \left(A \cdot T_{0}\right)\left[X_{k}+D\right]-\exp \left(T_{0}-\tau_{k}\right) D, \\
k=0,1,2,3, \ldots
\end{gathered}
$$

Here $\tau_{k}$

$$
\tau_{k}=\left\{\begin{array}{cl}
0, & \varepsilon_{k}<0 ; \\
T_{0}, & \varepsilon_{k}>V_{s} ; \\
\frac{T_{0} \varepsilon_{k}}{V_{s}}, & 0 \leq \varepsilon_{k} \leq V_{s} ;
\end{array}\right.
$$

with

$$
\varepsilon_{k}=\alpha\left(V_{r e f}-C X_{k}\right)
$$

where $C=(\beta, 0)$ is the row matrix.

In the following simulations, we shall use: $T_{1} T_{2}=10240 \mathrm{~s}^{2} ; T_{1}+T_{2}=352 \mathrm{~s} ; K$ $=327.8^{\circ} \mathrm{C} / \mathrm{V} ; T_{0}=10 \mathrm{~s} ; U=24 \mathrm{~V} ; \beta=0.01 \mathrm{~V} /{ }^{\circ} \mathrm{C} ; V s=5 \mathrm{~V} ; \alpha>0 ;$ Vref $=5 \mathrm{~V}[9,10]$.

The gain factor $\alpha$ and the input voltage $U_{0}$ will be used as the main control parameters. The period $T$ of periodic motion is an integer multiple to the rump period $T_{0}$, i.e. $T=m T_{0}$, $m=1,2, \ldots$ We will refer to this type motion as a period- $m$ cycle or $m-$ cycle.

\section{Bifurcation analysis}

At carrying out bifurcation analysis the supply voltage $U_{0}$ and amplification factor $\alpha$ have been selected as varying parameters. Depending on the parameter values, a variety of different bifurcation scenarios is observed. In Fig. 2 the one-parameter bifurcation diagrams, calculated for various values of $U_{0}$ at the alteration of the amplification factor $\alpha$, are presented.

At low values of $U_{0}$ the system demonstrates quasi-periodic behaviour with the pronounced multistablilty. In Fig. 2 (a) bifurcation diagram is shown, which illustrates the generation of a closed invariant curve, corresponding to the double-frequency quasiperiodic mode. As follows from Fig. 2 (a), when the amplification factor $\alpha$ increases, the 1-cycle becomes unstable through Neimark-Sacker bifurcation $[11,12]$. This results in the generation of a stable closed invariant curve, at which the 1-cycle still exists, but becomes an unstable focus. As it is known, the motion pattern on a closed invariant curve depends on the rotation number; if it is irrational, the invariant curve is densely filled with points of the map and the dynamics becomes quasiperiodic.

When the rotation number is rational, the invariant curve has an even number of periodic orbits, half of which are stable, and the others are saddle, and the invariant curve itself is composed of the unstable manifolds of saddle cycles. In Fig. 2 (a) the periodical dynamics window corresponds to the stability area of a resonant 4-cycle (the existence domain of a closed invariant curve with the rotation number 1:4). At the increase of $\alpha$ the resonant 4-cycle undergoes the period-doubling bifurcation cascade, which results in the chaotization of oscillations, at which the closed invariant curve is destroyed. The numerical experiments have shown that the stability area of 1-cycle is decreased with the increase of $U_{0}$. 




(a)

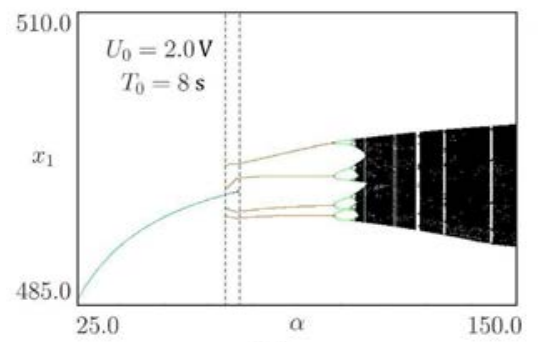

(c)

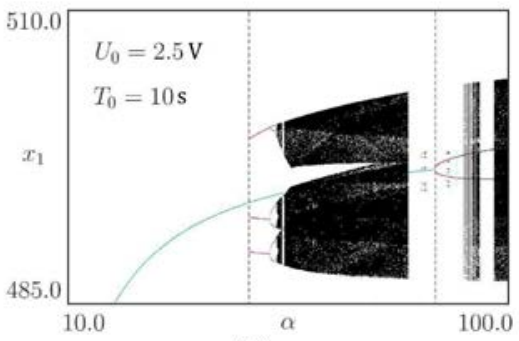

(b)

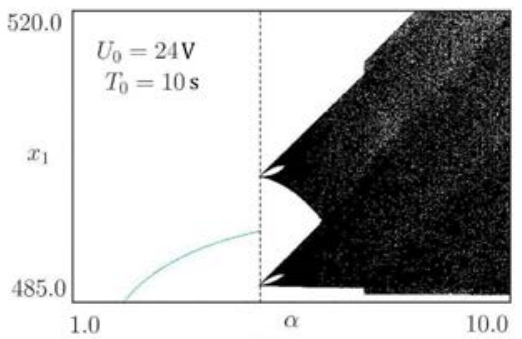

(d)

Fig. 2. Bifurcation diagrams at various parameters $U_{0}$ and $T_{0}, V_{s}=5 \mathrm{~V}, \mathrm{Vref}=5 \mathrm{~V}$ (corresponds to the preset $500^{\circ} \mathrm{C}$ )

The bifurcation diagram, presented in Fig. 2 (b), illustrates the typical scenario of coexisting attractors' appearance. At the alteration of amplification factor $\alpha$ there is a hard onset of a stable 3-cycle. At the further increase of $\alpha$ an infinite period-doubling bifurcation cascade takes place, resulting in chaotization of oscillations. For this reason, in the wide range of parameters variation along with the stable 1-cycle either the stable periodic oscillations or chaotic modes exist as well. So, depending on the initial conditions either periodical or chaotic motion can be set.

In Fig. 2 (c) an example of subcritical period quadrupling of the 1-cycle through the socalled «border-collision bifurcation», see example [8-14], is shown.

In Fig. 2 (d) an example of four-band chaotic attractor generation through bordercollision flip bifurcation $[8,14]$ is demonstrated.

\section{Conclusions}

The purpose of this paper was to study some of the complex dynamic phenomena that can arise in a high-power heating unit with pulse width modulated control. The mathematical model of this system was represented as a two-dimensional set of nonautonomous differential equations with discontinuous right-hand sides.

The first step in our investigation was to reduce this system to a two-dimensional piecewise-smooth map (7). It has been found out that at low values of supply voltage the system demonstrates quasi-periodic behaviour with the pronounced multistablilty [11], though the system has a large stability margin in the amplification factor at the same time. Nevertheless, with the increase of supply voltage the stability area of the 1-cycle (operation mode) is reduced, and the stability is lost through border-collision bifurcation, which results in the abrupt chaotization of oscillations.

In this paper, we discussed only the first steps towards understanding of the observed nonlinear phenomena. 
Further development of these investigations is important both from the theoretical and applied points of view, as it will provide a theoretical foundation for design and control of a wide class of pulse-modulated control systems.

The authors would like to thank Prof. Zh.T. Zhusubaliyev for his careful reading and constructive suggestions to the first draft of this manuscript.

The article was prepared within a development program of the Flagship University on the basis of BSTU named after V. G. Shukhov.

\section{References}

1. H. J. Scheel, T. Fukuda, Crystal growth technology (John Wiley \& Sons, 2003)

2. H. J. Scheel, P. Capper, Crystal growth technology: From fundamentals and simulation to large-scale production (John Wiley \& Sons, 2008)

3. Y.A. Gol’tsov, A.S. Kizhuk, V.G.Rubanov, Int. J. Pharm. Techn. 12, (2016)

4. V.G.Rubanov, A.S. Kizhuk, Y.A. Gol’tsov, Int. J. Soft Comp. 10, (2015)

5. M.H. Rashid, F.L. Luo, Power Electronics Handbook (Elsevier Science, 2011)

6. Zh.T. Zhusubaliyev, E. Mosekilde, Bifurcations and chaos in piecewise-smooth dynamical system (World Scientific, Singapore, 2003)

7. S.Banerjee, G. C. Verghese, Nonlinear phenomena in power electronics (IEEE Press, New York, 2001)

8. Di. M Bernardo, C. J. Budd, A. R. Champneys, P. Kowalczyk, Piecewise-smooth dynamical systems: Theory and applications (Springer-Verlag, New York, 2008)

9. Y.A. Gol'tsov, A.S. Kizhuk, and V.G.Rubanov, MATEC Web of Conf. 129, 01031 (2017)

10. Yu.A. Gol'tsov et al, IOP Conf. Ser.: Mater. Sci. Eng. 327, 052014 (2018)

11. J.M.T Thompson, H.B. Stewart, Nonlinear dynamics and chaos (Wiley, Chichester, 1986)

12. Yu.A. Kuznetsov, Elements of applied bifurcation theory (Springer-Verlag, New York, 2004)

13. Zh.T. Zhusubaliyev, E. Mosekilde, O.O Yanochkina, IEEE Trans. Power Electron. 26, $4(2011)$

14. Zh.T. Zhusubaliyev, E. Mosekilde, Math. Comput. Simul. 109 (2015) 